\title{
MET and PTEN gene copy numbers and Ki-67 protein expression associate with pathologic complete response in ERBB2- positive breast carcinoma patients treated with neoadjuvant trastuzumab-based therapy
}

Benjamin C. Calhoun ${ }^{1}$, Bryce Portier ${ }^{1,3}$, Zhen Wang ${ }^{1}$, Eugen C. Minca ${ }^{1}$, G. Thomas Budd ${ }^{2}$, Christopher Lanigan', Raymond R. Tubbs ${ }^{1}$ and Larry E. Morrison ${ }^{3 *}$

\begin{abstract}
Background: Pathologic complete response ( $p C R$ ) after neoadjuvant chemotherapy for breast cancer is associated with improved prognosis in aggressive tumor subtypes, including ERBB2- positive tumors. Recent adoption of pCR as a surrogate endpoint for clinical trials in early stage breast cancer in the neoadjuvant setting highlights the need for biomarkers that, alone or in combination, help predict the likelihood of response to treatment.

Methods: Biopsy specimens from 29 patients with invasive ductal carcinoma treated with trastuzumab-based therapy prior to definitive resection and pathologic staging were evaluated by dual color bright field in situ hybridization (dual ISH) using probes for MET, TOP2A, PTEN, and PIK3CA genes, each paired with centromeric probes to their respective chromosomes (chromosomes 7, 17, 10, and 3). Ki-67 expression was assessed by immunohistochemistry (IHC). Various parameters describing copy number alterations were evaluated for each gene and centromere probe to identify the optimal parameters for clinical relevance. Combinations of ISH parameters and IHC expression for Ki-67 were also evaluated.
\end{abstract}

Results: Of the four genes and their respective chromosomes evaluated by ISH, two gene copy number parameters provided statistically significant associations with PCR: MET gain or loss relative to chromosome 7 (AUC $=0.791$, sensitivity $=92 \%$ and specificity $=67 \%$ at optimal cutoff, $p=0.0032$ ) and gain of PTEN (AUC $=0.674$, sensitivity = $38 \%$ and specificity $=100 \%$ at optimal cutoff, $p=0.039$ ). Ki-67 expression was also found to associate significantly with $\mathrm{pCR}$ ( $A \cup C=0.726$, sensitivity $=100 \%$ and specificity $=42 \%$ at optimal cutoff, $p=0.0098$ ). Combining gain or loss of MET relative to chromosome 7 with Ki-67 expression further improved the association with pCR (AUC $=0.847$, sensitivity $=92 \%$ and specificity $=83 \%$ at optimal cutoffs, $p=0.0006$ ).

Conclusions: An immunogenotypic signature of low complexity comprising MET relative copy number and Ki-67 expression generated by dual ISH and IHC may help predict pCR in ERBB2-positive breast cancer treated with neoadjuvant chemotherapy and trastuzumab. These findings require validation in additional patient cohorts.

\footnotetext{
*Correspondence: Larry.morrison@roche.com

${ }^{3}$ Present Address: Ventana Medical Systems, Inc, 1910 E. Innovation Park Dr, Tucson, AZ 85755, USA

Full list of author information is available at the end of the article
} 
(Continued from previous page)

Keywords: Breast cancer, Neoadjuvant, Biomarkers, ERBB2, HER2, Pathologic complete response (pCR), In situ hybridization (ISH)

Abbreviations: ASCO, American society of clinical oncology; AUC, Area under curve; CAP, College of American pathologists; CEN, Centromere; CISH, Chromogen in situ hybridization; FDA, Food and drug administration; FFPE, Formalin fixed paraffin embedded; FISH, Fluorescence in situ hybridization; IHC, Immunohistochemistry; ISH, In situ hybridization; NSABP, National surgical adjuvant breast and bowel project; P, Probability; pCR, Pathologic complete response; ROC, Receiver operating characteristic; SISH, Silver in situ hybridization

\section{Background}

Pathologic complete response (pCR) after neoadjuvant chemotherapy for breast cancer is associated with improved prognosis [1]. The prognostic value of a pCR may be greatest in aggressive tumor subtypes, including ERBB2-positive tumors [1]. The Food and Drug Administration (FDA) has issued guidance on the use of pCR as a surrogate endpoint for clinical trials in early stage breast cancer in the neoadjuvant setting [2]. Pertuzumab, an inhibitor of heterodimerization of ERBB2 (erb-b2 receptor tyrosine kinase 2, commonly known as HER-2 and HER2/neu) with other ERBB receptor family members, is the first agent granted accelerated approval for the neoadjuvant treatment of high-risk early stage breast cancer based on pCR data [3]. Given the importance of pCR in prognosis and clinical trial design, there is a need to identify biomarkers that, alone or in combination, help predict the likelihood of response to treatment. A variety of genes, including PIK3CA, PTEN, TOP $2 A$ and $M E T$ are candidate markers for prognosis and response to treatment in ERBB2-positive breast cancer.

Genetic alterations in the phosphatidylinositol 3-kinase (PI3K)/V-AKT murine thymoma viral oncogene homolog $(\mathrm{AKT}) /$ mechanistic target of rapamycin (MTOR) pathway are common events in breast cancer $[4,5]$. Preclinical data in cell lines indicate that mutations in the p110 alphacatalytic subunit of PI3K (PIK3CA) lead to resistance to trastuzumab and lapatinib [6-9]. Several clinical studies have examined the association between somatic mutations in PIK3CA and benefit from ERBB2-targeted therapy [1014]. In the FinHer [10] and NSABP B-31 adjuvant trials [11], there was no significant loss of trastuzumab efficacy observed in patients with PIK3CA mutations. In the NeoALLTO neoadjuvant trial which incorporated lapatinib as well as trastuzumab, patients with PIK3CA mutations were less likely to have a pCR, but there were no significant differences in progression-free or overall survival [12]. Other neoadjuvant trials have shown similar results [13, 14]. Comparatively little is known about PIK3CA gene copy number alterations and clinical outcomes in breast cancer, irrespective of ERBB2 status [15]. Amplification of mutant PIK3CA alleles appears to contribute to resistance to PI3K inhibitors in preclinical breast cancer models [16].
Phosphatase and tensin homolog (PTEN) is the 3' lipid phosphatase for phosphatidylinositol-3,4,5-triphospate (PIP3), thereby negatively regulating downstream signaling by PIP3 after phosphorylation by PI3K $[17,18]$. Patients with ERBB2-positive, PTEN-deficient tumors may develop resistance to ERBB2-targeted therapy [6, 9, 19-22]. Many small, retrospective studies have shown that PTEN deficiency or absence may be associated with reduced clinical benefit from trastuzumab [19, 20, 23, 24]. However, the recent data from a large prospective study of early stage ERBB2-positive breast cancer indicate that patients with and without PTEN deficiency by immunohistochemistry derived benefit from treatment with trastuzumab [25]. PTEN status in most studies was determined by immunohistochemistry or gene sequencing and relatively little is known about the significance of PTEN copy number alterations in response to ERBB2-targeted therapy.

The topoisomerase II alpha (TOP2A) and ERBB2 genes are located close to each other on the long arm of chromosome 17 and may be co-amplified in breast cancer [26-29]. Approximately $35 \%$ of ERBB2-amplified tumors show TOP $2 A$ gene amplification $[30,31]$ and deletions are much less common [30, 32]. Alterations in TOP $2 A$ copy number have mainly been associated with response to anthracycline-based chemotherapy [30,33]. The role of TOP2A amplification or deletion in response to ERBB2targeting has not been thoroughly investigated.

Overexpression of MET proto-oncogene, receptor tyrosine kinase (MET) occurs in $20 \%-30 \%$ of invasive breast cancers [34] and is associated with a poor prognosis in lymph node-positive and lymph node-negative disease and across all molecular subtypes [35-40]. In the metastatic setting, increased MET copy numbers correlate with trastuzumab therapy failure in ERBB2-positive breast cancer [41] and clinical trials with anti-MET therapy in advanced breast cancer are ongoing [42]. The significance of MET amplification or deletion in the response to adjuvant or neoadjuvant therapy for ERBB2-positive breast cancer is not well established.

In this exploratory study using in situ hybridization (ISH) and immunohistochemistry (IHC), we assessed alterations in gene copy number for PIK3CA, PTEN, $T O P 2 A$ and $M E T$, and their respective chromosomes, 
and expression of Ki-67 in a series of patients with ERBB2-positive tumors who were treated with chemotherapy and trastuzumab in the neoadjuvant setting. Various parameters representing gene and chromosome copy numbers were evaluated for association with $\mathrm{pCR}$ in an effort to identify parameters most effective for improving prediction in the neoadjuvant treatment of ERBB2-positive breast cancer.

\section{Methods}

This study was approved by the Cleveland Clinic Institutional Review Board. All patients who received trastuzumab at the Cleveland Clinic from January 2008 to
December 2010 were reviewed for study inclusion (234 patients). Of the 234 cases, 29 satisfied inclusion criteria which included a diagnosis of primary invasive breast cancer, neoadjuvant trastuzumab therapy, and a pretreatment biopsy performed at the Cleveland Clinic. Pathology data was obtained from the Anatomic Pathology information system CoPath Plus (Cerner Corporation, Kansas City, MO). Clinical data was obtained from the electronic medical record Epic (Epic Systems Corporation, Verona, WI). The age, tumor size, pre-treatment clinical stage, hormone receptor status by IHC, ERBB2 status by ISH, post-treatment pathologic stage, and presence or absence of a pCR were recorded for all patients (Table 1).

Table 1 Clinical and pathologic characteristics of patients with ERBB2-positive breast cancer treated with neoadjuvant chemotherapy and trastuzumab

\begin{tabular}{|c|c|c|c|c|c|c|c|c|c|}
\hline Case ID & Size, largest (mm) & Clinical TNM & Clinical stage & $\mathrm{ER} I \mathrm{HC}$ & $\mathrm{PR} I \mathrm{HC}$ & HER2 copy number (Average) & HER2/CEP17 Ratio & Pathologic Stage & $\mathrm{pCR}$ \\
\hline 1 & 49 & $\mathrm{CT} 2 \mathrm{~N} 2 \mathrm{MO}$ & IIIA & pos & pos & 9.1 & 4.1 & ypTONO & yes \\
\hline 2 & 47 & CT2NOMO & $\| \mathrm{A}$ & neg & neg & $7.4^{c}$ & $3.7^{c}$ & ypTisNo & yes \\
\hline 3 & 37 & cT2NOMO & $\| \mathrm{A}$ & neg & neg & 18.5 & 8.4 & ypTisNo & yes \\
\hline 4 & 12 & cT1N1M0 & $\| \mathrm{A}$ & pos & neg & 19.2 & 9.6 & ypTONO & yes \\
\hline 5 & 60 & cT3N1M0 & $\| I I A$ & pos & neg & 8.1 & 3.7 & ypTisNo & yes \\
\hline 6 & 63 & cT3N1M0 & $\| \mathrm{A}$ & neg & neg & 20.0 & 11.1 & ypTONO & yes \\
\hline 7 & 20 & cT4N1M0 & $\| \mathrm{IIB}$ & neg & neg & 17.2 & 6.6 & ypTONO & yes \\
\hline 8 & 36 & CT2N1M0 & $\| B$ & neg $^{a}$ & neg $^{a}$ & 17.4 & 6.7 & ypTONO & yes \\
\hline 9 & 18 & $\mathrm{CT} 1 \mathrm{~N} 2 \mathrm{M} 0$ & $\| I I A$ & pos & pos & 11.3 & 4.5 & ypTONO & yes \\
\hline 10 & 28 & cT4N1M0 & $\| I B$ & pos & pos & 15.8 & 7.5 & ypTONO & yes \\
\hline 11 & 47 & CT2NOMO & $\| \mathrm{A}$ & neg $^{a}$ & neg $^{a}$ & 4.3 & 2.9 & ypTONx & yes \\
\hline 12 & 36 & cT2NOMO & $\| \mathrm{A}$ & neg & neg & 13.2 & 5.5 & ypTONO & yes \\
\hline 13 & 37 & cT4NOMO & $\| I \mid B$ & pos & pos & 20.0 & 16.7 & ypTONO & yes \\
\hline 14 & 42 & cT2N1M0 & $\| \mathrm{B}$ & pos & neg & 3.3 & 2.4 & ypTONO & yes \\
\hline 15 & 60 & cT3NOMO & $\| \mathrm{B}$ & pos & pos & 14.7 & 8.2 & ypTONO & yes \\
\hline 16 & 20 & cT1N1M0 & $\| \mathrm{A}$ & pos & pos & 4.9 & 3.1 & ypTisN1 & no \\
\hline 17 & 60 & cT4N1M0 & $\| I \mathrm{~B}$ & pos & pos & 5.0 & 3.1 & ypT3No & no \\
\hline 18 & 64 & cT3NOMO & $\| B$ & pos & pos & 16.1 & 14.6 & ypT1miNo & no \\
\hline 19 & 51 & cT3N1M0 & $\| \mathrm{A}$ & pos & pos & 5.2 & 3.2 & ypT1No & no \\
\hline 20 & 40 & cT2N1M0 & $\| I I A$ & pos & pos & 4.4 & 2.3 & ypT2N1 & no \\
\hline 21 & 21 & CT2N1M0 & $\| \mathrm{A} A$ & pos & pos & 16.6 & 8.3 & ypT1No & no \\
\hline 22 & 35 & CT4N1M0 & $\| \mathrm{II}$ & pos & $n_{e g}{ }^{a}$ & 5.3 & 2.9 & ypT3NO & no \\
\hline 23 & 86 & cT3N1M0 & $\| I I A$ & pos & pos & 5.0 & 3.2 & ypT1N1 & no \\
\hline 24 & 27 & cT2N1M0 & $\| I I A$ & neg & neg & 15.9 & 7.2 & ypT1N2 & no \\
\hline 25 & 43 & cT2N1M0 & \|\| $\mathrm{A}$ & pos & pos & 14.0 & 5.6 & ypT1No & no \\
\hline 26 & 60 & cT4N2M0 & $\| \mathrm{II}$ & pos & pos & 16.7 & 8.4 & урT3N1 & no \\
\hline 27 & 29 & cT2N2M0 & $\| I I A$ & pos & pos & 16.2 & 10.1 & ypT1N2 & no \\
\hline 28 & 73 & cT3NOMO & $\| \mathrm{B}$ & pos & pos & 9.1 & 3.5 & ypT2N1 & no \\
\hline 29 & 41 & CT2NOMO & $\| \mathrm{A}$ & neg & neg & $3.6^{\mathrm{b}}$ & $1.8^{\mathrm{b}}$ & ypT1N1 & no \\
\hline
\end{tabular}

Abbreviations: $E R$ estrogen receptor, $I H C$ immunohistochemistry, $P R$ progesterone receptor, $p C R$ pathologic complete response

${ }^{a}$ Cases reported as negative, $<5 \%$, prior to the 2010 ASCO/CAP Guidelines

${ }^{b}$ ERBB2 immunohistochemistry was $3+$

CERBB2 genetic heterogeneity present; average ERBB2 copy number and ERBB2/CEP17 ratio reported for amplified clone 


\section{Patients and clinical assessment}

Formalin-fixed paraffin-embedded (FFPE) needle biopsy specimens from early stage breast cancer patients treated in the neoadjuvant setting were obtained from the archives of the Cleveland Clinic (Cleveland, $\mathrm{OH}$ ). Chart review and study analyses were approved by the Cleveland Clinic institutional review board. Eligibility criteria for further evaluation included histologic confirmation of clinical stage IIA to IIIB, ERBB2 amplification by in fluorescent in situ hybridization (FISH), and neoadjuvant treatment that included trastuzumab. Post-treatment pathologic staging was obtained from pathology reports and confirmed by histologic evaluation. For this study, classification as $\mathrm{PCR}$ required the absence of any detectable invasive carcinoma in the breast specimen and the axillary lymph nodes (i.e., ypTONO and ypTisN0).

With respect to treatment, of the 29 patients in the cohort 11 were treated with anthracycline-based chemotherapy including cyclophosphamide, a taxane, and trastuzumab (i.e. ACTH) and 18 received a taxane and trastuzumab with or without carboplatin (TCH). Of the 15 patients with a pCR, 5 were treated with $\mathrm{ACTH}$ and 10 were given $\mathrm{TCH}$. Of the 14 patients who did not have a pCR, 6 received ACTH and 8 were treated with TCH. A total of 12 patients presented with clinical stage IIA or IIB disease and 8 of these patients had a pCR. Of the 8 patients with a pCR, 3 were treated with ACTH and 5 were given $\mathrm{TCH}$. Of the 4 stage II patients who did not have a pCR, 1 was treated with $\mathrm{ACTH}$ and 3 were given $\mathrm{ACTH}$. In this small exploratory study there may be some imbalance in the distribution of stage II patients in the two treatment groups. However, we believe the distribution of patients treated with $\mathrm{ACTH}$ versus $\mathrm{TCH}$ is relatively well-balanced among those who did or did not have a pCR.

\section{Immunohistochemistry}

Ki-67 automated IHC was performed on 3-6 $\mu$ m thick sections of FFPE specimen blocks using primary antibody 30-9 with iVIEW detection on the VENTANA BenchMark $\mathrm{XT}$ automated stainer instrument (all reagents and instrument from Ventana Medical Systems, Tucson, AZ) using the company recommended protocols.

\section{Silver and chromogenic in situ hybridization (SISH and CISH)} Automated in situ hybridization (ISH) was performed on 3-6 $\mu \mathrm{m}$ thick sections of FFPE specimen blocks using the Ventana Medical Systems dual ISH procedure (dual color dual hapten DNA in situ hybridization) on the BenchMark XT automated stainer. Probes were hybridized in the following pairs, each comprising a gene locus probe, referred to by the name of a gene contained within the targeted region (e.g. MET), and a centromere (CEN) probe for the chromosome on which the gene locus lies (e.g. CEN7): $M E T+$ CEN7, TOP2A + CEN17, $P T E N+$ CEN10, and PIK3CA + CEN3. All gene probes were detected using peroxidase-catalyzed silver staining (SISH) and centromeric probes were detected using chromosome staining with alkaline phosphatasecatalyzed fast red staining (CISH). ISH probes and associated detection reagents are commercially available from Ventana Medical Systems.

\section{Fluorescent in situ hybridization (FISH)}

$E R B B 2$ status determination was performed using an FDA-approved interphase FISH assay (PathVysion ${ }^{\circ}$, Abbott Molecular, Des Plaines, IL). Consistent with the timeframe in which the patients were treated, ERBB2 scoring methods were applied to FISH samples in accordance with the 2007 ASCOCAP guidelines [43]. Briefly, ASCOCAP dual-probe scoring was applied as follows: non-amplified $(E R B B 2 C E P 17<1.8)$, equivocal (ERBB2CEP17 1.8-2.2), or amplified (ERBB2CEP17 > 2.2). For the cases in which it was performed, ERBB2 IHC (4B5, Ventana Medical Systems Inc, Tucson, AZ) was scored according to the 2007 ASCOCAP guidelines [43] as $0,1,2$, or 3 .

\section{Specimen evaluation}

Hybridized and coverslipped specimens were viewed with brightfield microscopy to enumerate the SISH (metallic silver - black) gene locus signals and CISH (red) centromere signals on a cell-by-cell basis. Only invasive carcinoma tumor cells were selected for cellby-cell signal enumeration using a $40 \mathrm{X}$ objective in combination with $10 \mathrm{X}$ eyepieces. In general, 50 invasive tumor cells were enumerated per specimen except in several specimens for which fewer than 50 cells with good hybridization signals could be found (a minimum of 20 cells were required for inclusion in the analysis). Gene locus and centromere copy number statuses were assessed using a number of different parameters, including:

1) the average number of gene or centromere copies per cell, designated as gene/cell (e.g. MET/cell) or centromere/cell (e.g. CEN7/cell),

2) the percentage of cells with greater than 2 gene or centromere signals, designated as gene gain (e.g. $M E T$ gain) or centromere gain (e.g. CEN7 gain),

3) the percentage of cells with less than 2 gene or centromere signals, designated as gene loss (e.g. $M E T$ loss) or centromere loss (CEN7 loss),

4) the percentage of cells with either greater than 2 or less than 2 gene locus or centromere signals, designated as gene gain or loss (e.g. MET gain or loss), or centromere gain or loss (e.g. CEN7 gain or loss), 
5) the average number of gene copies per corresponding centromere copies, designated as gene locus/centromere (e.g. MET/CEN7),

6) the percentage of cells with more copies of the gene than the corresponding centromere, designated as gene/centromere gain (e.g. MET/CEN7 gain),

7) the percentage of cells with fewer copies of the gene than the corresponding centromere, designated as gene/centromere loss (e.g. MET/CEN7 loss), and

8) the percentage of cells with either more copies of the gene than the corresponding centromere or fewer copies of the gene than the corresponding centromere, designated as gene/centromere gain or loss (e.g. $M E T / C E N 7$ gain or loss).

The average number of genes or centromeres per cell was calculated by summing the number of gene or centromere signals over all the cells enumerated within a specimen, and dividing by the number of cells enumerated. The average number of genes per centromere was calculated by summing the number of gene signals over all the cells enumerated and dividing by the sum of the centromere signals in all of the cells enumerated.

For Ki-67 IHC staining interpretation was performed only for invasive tumor cells and was evaluated by selecting the area of invasive carcinoma with the highest proliferation rate and then determining the percentage of approximately 50 invasive tumor cell nuclei that were positive for Ki-67 expression. The cutoff for Ki-67 positively staining cells was determined empirically to provide the best combined sensitivity and specificity for $\mathrm{PCR}$ in this neoadjuvant setting. An additional data file (.xls) lists the values for each ISH and IHC parameter described above for each patient in the study [Additional file 1].

For each parameter a range of cutoffs was evaluated such that specimens with the parameter value greater than or equal to a cutoff were considered 'high' for that parameter and specimens with the parameter value less than the cutoff were considered 'low' for that parameter. Sensitivities and specificities for detecting patients with $\mathrm{pCR}$, based on either the high parameter being positive for $\mathrm{pCR}$ or based on the low parameter being positive for $\mathrm{pCR}$, were calculated for each parameter and each cutoff. Receiver Operating Characteristics (ROC) curves were generated as sensitivity versus 1 - specificity over all cutoffs tested, and Area Under the Curve (AUC) was calculated for each curve as one measure of a parameter's ability to distinguish patients with $\mathrm{pCR}$ from patients without $\mathrm{pCR}$, with $\mathrm{AUC}=1$ being ideal and progressively lower values being less favorable. AUC values near 0.5 indicate no ability to distinguish between patients. In addition to ROC analysis, 2X2 contingency tables were evaluated at each cutoff and probabilities from Fischer's Exact test were used to gauge the statistical significance of the association between the binarized parameter (high versus low values) and pCR. The optimal cutoff for a parameter was the cutoff value providing the best combined sensitivity and specificity, which typically provided the lowest $p$-value.

In addition to the various single parameters, a ROC curve for the combinations of MET/CEN7 gain or loss with Ki-67 expression was generated by using the cutoff providing optimal sensitivity and specificity for $M E T /$ CEN7 gain or loss while varying the cutoff for Ki-67 over a wide range, with the combined parameters considered positive if both parameters were equal to or greater than the respective cutoff values, as described by Shultz, 1995 [44].

\section{Results}

A total of 29 patients meeting inclusion criteria with tissue available for IHC and ISH studies were identified (Table 1). Of these patients, 27 had Ki-67 expression data, 24 had MET and CEN7 ISH counts, 25 had PTEN and CEN10 ISH counts, and 24 had both Ki-67 expression data and MET and CEN7 ISH counts. The mean and median age at presentation was 53 and 52 years, respectively. The mean and median pre-treatment tumor size was 49 and $41 \mathrm{~mm}$, respectively (range $=12-86$ ). Patients who presented with Stage IV disease and who underwent breast surgery were excluded. Overall, 15 of $29(52 \%)$ patients had a pCR, defined as ypT0/ypTis NO. Among the 14 patients who did not have a pCR, the residual invasive tumor measured less than $1 \mathrm{~mm}$ in 1 patient and 1 patient had residual ductal carcinoma in situ (DCIS) and a positive lymph node (ypTisN1).

Gene copy numbers for TOP2A, MET, PTEN, and PIK3CA, and their corresponding chromosome copy numbers were measured by ISH, and $\mathrm{Ki}-67$ protein expression was measured by IHC to identify associations with $\mathrm{pCR}$ individually and in combination. Figure 1, parts $\mathrm{A}, \mathrm{B}$, and $\mathrm{C}$, show representative Ki-67 IHC, $M E T$ (black) + CEN7 (red) ISH, and PTEN (black) + CEN10 (red) ISH staining, respectively, on selected FFPE sections of biopsy specimens from the neoadjuvant breast cancer cohort. Figure 1a shows Case \#2 (see Table 1 for characteristics of case \#2) stained by IHC for Ki-67 and was determined to express Ki-67 in greater than $90 \%$ of the cells. Figure $1 \mathrm{~b}$ shows Case \#2 stained for MET (black) and CEN7 (red) by ISH and shows cells with a lesser number of MET signals than CEN7 signals (48\% of cells showed relative $M E T$ loss). The tumor had other areas with a greater number of $M E T$ signals than CEN7 signals (34 \% of cells showed relative $M E T$ gain) as well. Figure 1c shows Case \#3 stained for PTEN (black) and CEN10 (red) with increased copy numbers for both loci (86\% of cells are near-tetrasomy). 


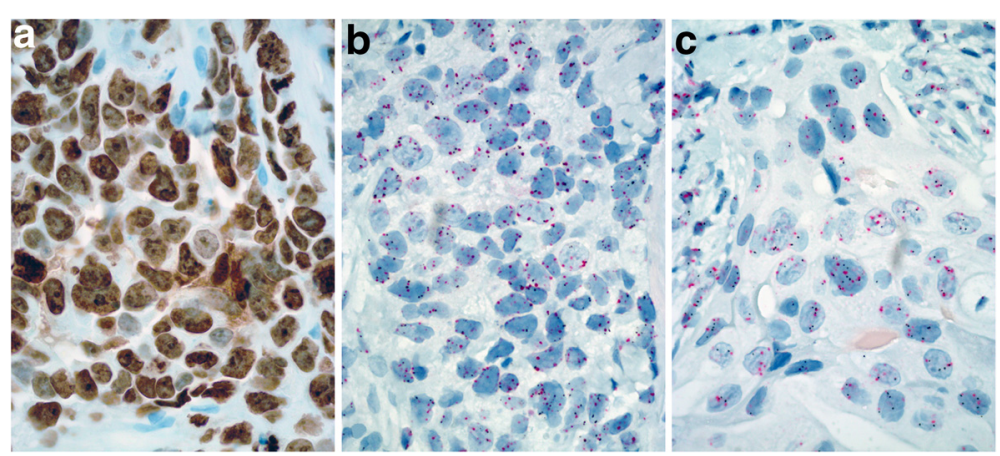

Fig. 1 Representative images of immunohistochemistry and in situ hybridization studies from three tumors. a: Immunohistochemistry for Ki-67 showing positive staining in greater than $90 \%$ of nuclei in a specimen from Case \#2. b: ISH for MET + CEN7 showing reduced MET copy number [silver (black) signals] relative to chromosome 7 [red signals] in a specimen Case \#2. c: ISH for PTEN + CEN10 showing gains in PTEN copy number [silver (black) signals] and chromosome 10 copy number [red signals] in a specimen from Case \#3. (Original magnification x 600)

ISH signals were interpreted using several different parameters to express gene and chromosome copy numbers in order to determine which parameters best associated with pCR. Figure 2 shows plots of 5 ROC curves, each representing a different parameter describing $M E T$ gene copy number relative to chromosome 7 copy number (see Methods section for a definition of each parameter). AUC values for each curve are listed in Table 2, along with the optimal cutoff values, whether the high parameter values (equal to or above the cutoff) or low parameter values (below the cutoff) were associated with pCR to generate the ROC curve and contingency analysis, the sensitivity

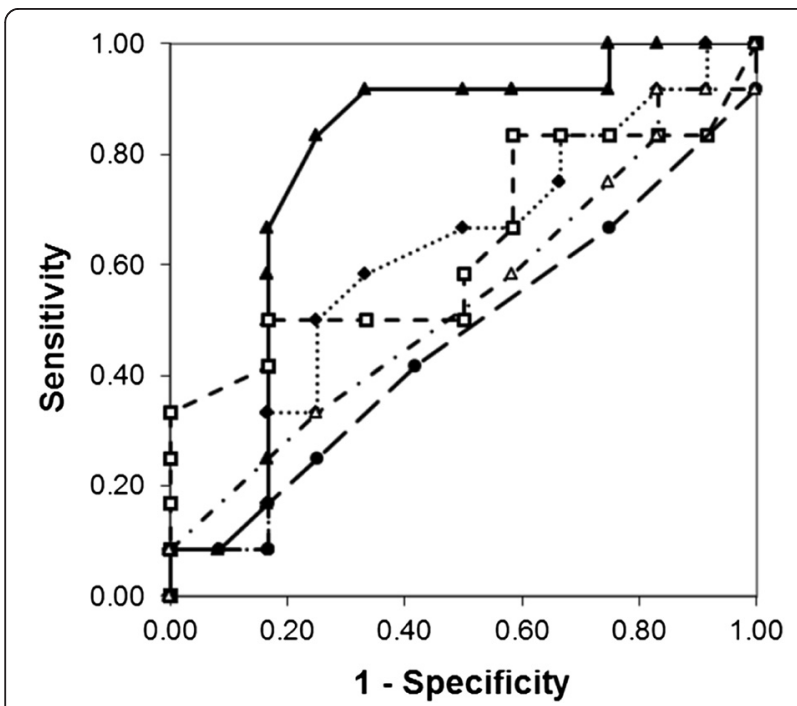

Fig. 2 ROC curves for different parameters measuring MET gene copy number relative to chromosome 7 copy number. Parameters plotted include: MET/CEN7 gain or loss (solid line with solid triangles), MET/CEN7 gain (dotted line with solid diamonds), MET/ CEN7 loss (short dashed line with open squares), MET/CEN7 (high ratios associated with $\mathrm{pCR}$; long dashed line with solid circles), and MET/CEN7 (low ratios associated with pCR; alternating dashed and dotted line with open triangles) and specificity obtained for those optimal cutoff values, and $p$-values using Fischer's Exact test for the 2x2 contingency tables generated at those cutoffs. These ROC curves show a large difference between the association of the various $M E T / C E N 7$ parameters and $\mathrm{pCR}$. The two ROC curves representing average $M E T / C E N 7$ counts, one associating ratios greater than the cutoff with $\mathrm{pCR}$ and the other associating ratios lower than the cutoff with $\mathrm{pCR}$, show little if any association (AUC values near 0.5 ). The parameters representing the percentages of cells with more copies of MET than CEN7 (MET/CEN7 gain) and the percentages of cells with less copies of $M E T$ than CEN7 (MET/CEN7 loss) provided larger AUC values (0.613 and 0.651, respectively), while the parameter based on the sum of the percentage of cells with more copies and less copies of MET than CEN7 (MET/CEN7 gain or loss) provided the largest AUC value (0.791).

Other parameters showing associations with $\mathrm{pCR}$ included Ki-67 expression (AUC $=0.726$ ) and the percentage of cells with greater than the normal 2 PTEN copies, $P T E N$ gain $(A U C=0.674)$, the ROC curves of which are plotted in Fig. 3, and whose AUC values, optimal cutoffs, related sensitivities and specificities, and $p$-values are included in Table 2. The parameter $M E T / C E N 7$ gain or loss was further analyzed in combination with Ki-67 expression, the 2 parameters providing the largest AUC values alone. This was done by generating an ROC curve [44] in which the cutoff for MET/CEN7 gain or loss was held at its optimal value of $50 \%$ cells while varying the cutoffs for Ki-67 expression (plotted in Fig. 3), requiring both parameters in the combination to be equal to or greater than their respective cutoffs for a positive designation. The additional AUC provided by the combination with Ki-67 was 0.056 over that of $M E T / C E N 7$ gain or loss alone. The optimal cutoff for Ki-67 expression in combination with MET/CEN7 gain or loss was $8 \%$ cells, maintaining the sensitivity at $92 \%$ while increasing the specificity from 67 to $83 \%$, and improving 
Table 2 Contingency table and ROC analysis results for parameter associations with $\mathrm{pCR}$

\begin{tabular}{|c|c|c|c|c|c|c|c|c|}
\hline Parameter & $N, p C R$ & $N$, non-pCR & pCR correlated state ${ }^{a}$ & ROC AUC & Optim c/o & Sens & Spec & $p$ \\
\hline MET/CEN7 gain or loss & 12 & 12 & high & 0.791 & $50 \%$ cells & 0.92 & 0.67 & 0.0094 \\
\hline MET/CEN7 gain & 12 & 12 & high & 0.613 & $32 \%$ cells & 0.58 & 0.67 & 0.41 \\
\hline MET/CEN7 losS & 12 & 12 & high & 0.651 & $24 \%$ cells & 0.50 & 0.83 & 0.19 \\
\hline MET/CEN7 & 12 & 12 & high & 0.479 & 1.10 & 0.42 & 0.58 & 1.0 \\
\hline MET/CEN7 & 12 & 12 & low & 0.547 & 1.00 & 0.33 & 0.75 & 1.0 \\
\hline Ki-67 & 15 & 12 & high & 726 & $8 \%$ cells & 1.00 & 0.42 & 0.0098 \\
\hline MET/CEN7 gain or loss AND Ki-67 & 12 & 12 & high/high & $847^{\mathrm{b}}$ & $50 \%$ cells/8 \% cells & 0.92 & 0.83 & 0.0006 \\
\hline PTEN gain & 13 & 12 & high & 674 & $58 \%$ cells & 0.38 & 1.00 & 0.039 \\
\hline
\end{tabular}

Abbreviations: $N$ number of specimens, $p C R$ pathologic complete response, AUC area under curve, Optim c/o optimal cutoff (cutoff producing best combined sensitivity and specificity), Sens sensitivity, Spec specificity, $p$ probability calculated using Fischer's Exact test on contingency tables generated using optimal cutoff(s) as executed using JMP Statistical Software (SAS, Cary, NC)

${ }^{a}$ The parameter state that is associated with $\mathrm{PCR}$ in ROC curve and contingency table calculations, for which high state comprises specimens with parameter values equal to or greater than the optimum cutoff and low state comprises specimens with parameter values less than the optimum cutoff

${ }^{\mathrm{b}}$ The AUC for the combined parameters equals the area under the ROC curve of MET/CEN7 gain or loss plus the additional area under the ROC curve of MET/CEN7 gain or loss, holding cutoff constant at $50 \%$, combined with Ki-67, varied across all possible cutoffs

the $p$-value to 0.0006 (see Table 2). PTEN copy number was also evaluated in combination with Ki-67 expression but improvement in $p$-value and combined sensitivity and specificity over the individual parameters was less (data not shown).

Clinical and pathologic characteristics listed in Table 1 for each patient were compared to the ISH and IHC parameters in Table 2 that showed statistically significant associations with $\mathrm{pCR}$, as well as compared to $\mathrm{pCR}$, using contingency tables (stage, ER IHC, PR IHC) and t-tests (age, tumor size, ERBB2/cell, and ERBB2/CEN17). Results are listed in Table 3 and show few statistically

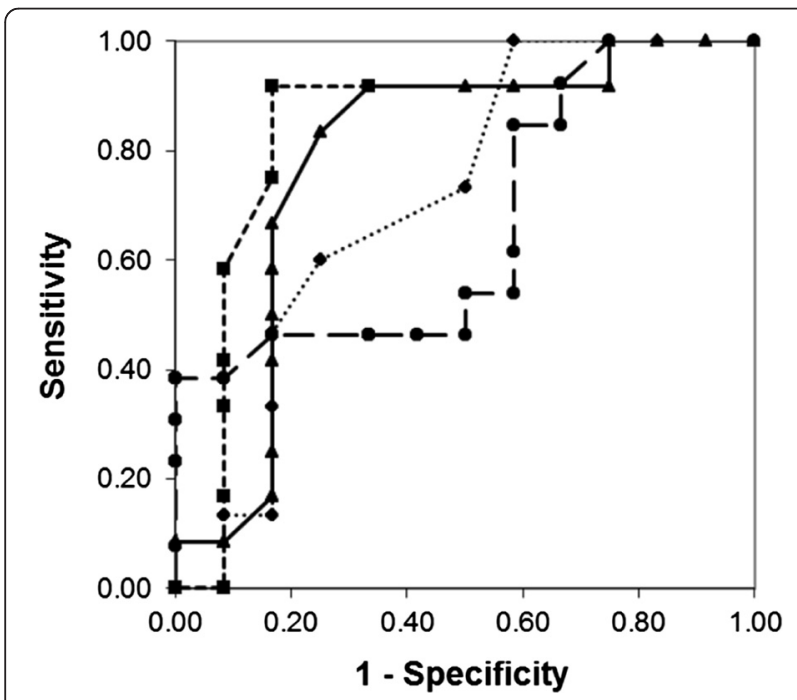

Fig. 3 ROC curves for MET, Ki-67, PTEN, and MET combined with Ki-67 parameters. Parameters plotted include: MET/CEN7 gain or loss (solid line with solid triangles; repeated from Fig. 2), Ki-67 expression (dotted line with solid diamonds), PTEN gain (long dashed line with solid circles), and MET/CEN7 gain or loss held at a constant cutoff of $50 \%$ of cells while varying the cutoff for Ki-67 expression (short dashed line with solid squares) significant associations. MET/CEN7 gain or loss and MET/CEN7 gain or loss combined with Ki-67 expression were significantly associated with age (trend with tumor size), Ki-67 expression was strongly associated with both $E R B B 2 /$ cell and $E R B B 2 / C E N 17$, and pCR was significantly associated with only PR expression (trends with ER expression, ERBB2/cell, and age).

\section{Discussion}

In an effort to better identify patients more likely to achieve pCR in patients with ERBB2-positive breast cancer, we have evaluated a series of additional gene and centromere probes as well as Ki-67 expression. In our cohort pCR was achieved in $52 \%$ of patients. As part of the analysis of ISH results we have evaluated different parameters for describing abnormal gene and chromosome copy numbers. This is because there is no single parameter that best describes copy number for all genes and chromosomes for all tumors. For example, gene amplification is often defined as the presence of two or more copies of a gene per copy of the chromosome on which the gene normally resides. This definition of gene amplification was found to have strong clinical relevance with respect to prognosis [45] in breast cancers but has been applied widely to other genes and other cancers with little or no justification. In the present study we have also evaluated the ratio of various gene copy numbers to their respective chromosome copy numbers (as represented by the centromere copy number) and evaluated a wide range of cutoff values. As another measure of relative gene copy number, the percentage of cells with more gene than chromosome copies (gene/centromere gain), or less gene than chromosome copies (gene/ centromere loss), or either more or less gene copies relative to their respective chromosomes (gene/centromere gain or loss) were evaluated. Additionally, we have 
Table 3 Associations between clinical and pathologic characteristics (Table 1) and ISH parameters and pCR

\begin{tabular}{|c|c|c|c|c|c|c|c|c|c|c|c|}
\hline \multirow[t]{2}{*}{ Parameter $^{a}$} & & \multirow{2}{*}{$\begin{array}{l}\text { Mean } \\
\text { age } \pm S D\end{array}$} & \multirow{2}{*}{$\begin{array}{l}\text { Mean size, } \\
\text { largest } \\
(\mathrm{mm}) \pm \mathrm{SD}\end{array}$} & \multicolumn{2}{|c|}{ Stage } & \multicolumn{2}{|c|}{ ER IHC } & \multicolumn{2}{|c|}{$\mathrm{PR} I \mathrm{HC}$} & \multirow{2}{*}{$\begin{array}{l}\text { Mean } \\
\text { ERBB2/cell }\end{array}$} & \multirow{2}{*}{$\begin{array}{l}\text { Mean } \\
\text { ERBB2/CEN17 }\end{array}$} \\
\hline & & & & $\|$ & III & pos & neg & pos & $\overline{\text { neg }}$ & & \\
\hline \multirow[t]{3}{*}{ MET/CEN7 gain or loss } & high & $59.3 \pm 13.4$ & $39.4 \pm 16.6$ & 8 & 7 & 9 & 6 & 5 & 10 & $10.6 \pm 5.8$ & $4.9 \pm 2.3$ \\
\hline & low & $47.0 \pm 13.0$ & $51.2 \pm 18.6$ & 3 & 6 & 7 & 2 & 7 & 2 & $11.9 \pm 5.6$ & $6.9 \pm 4.0$ \\
\hline & $p$ & 0.041 & 0.14 & 0.42 & & 0.66 & & 0.09 & & 0.59 & 0.19 \\
\hline \multirow[t]{3}{*}{ Ki-67 } & high & $54.8 \pm 14.8$ & $41.6 \pm 15.4$ & 9 & 13 & 14 & 8 & 11 & 11 & $13.1 \pm 5.5$ & $7.0 \pm 3.8$ \\
\hline & low & $46.0 \pm 10.7$ & $57.2 \pm 21.6$ & 2 & 3 & 4 & 1 & 3 & 2 & $5.6 \pm 2.0$ & $2.9 \pm 0.7$ \\
\hline & $\mathrm{p}$ & 0.16 & 0.19 & 1.00 & & 0.64 & & 1.00 & & $<0.0001$ & $<0.0001$ \\
\hline \multirow[t]{3}{*}{ MET/CEN7 gain or loss AND Ki-67 } & high & $61.5 \pm 12.9$ & $37.2 \pm 14.9$ & 7 & 6 & 7 & 6 & 4 & 9 & $11.2 \pm 6.0$ & $5.1 \pm 2.4$ \\
\hline & low & $46.5 \pm 11.8$ & $51.7 \pm 18.7$ & 4 & 7 & 9 & 2 & 8 & 3 & $11.1 \pm 5.4$ & $6.2 \pm 3.9$ \\
\hline & $\mathrm{p}$ & 0.0071 & 0.051 & 0.44 & & 0.210 & & 0.10 & & 0.97 & 0.43 \\
\hline \multirow[t]{3}{*}{ PTEN gain } & high & $55.6 \pm 10.1$ & $40.6 \pm 16.7$ & 2 & 3 & 2 & 3 & 2 & 3 & $15.3 \pm 4.8$ & $7.0 \pm 2.9$ \\
\hline & low & $49.3 \pm 14.1$ & $46.8 \pm 17.7$ & 9 & 11 & 15 & 5 & 13 & 7 & $10.3 \pm 5.7$ & $5.8 \pm 4.1$ \\
\hline & $\mathrm{p}$ & 0.28 & 0.49 & 1.00 & & 0.28 & & 0.36 & & 0.83 & 0.50 \\
\hline \multirow[t]{3}{*}{$\mathrm{pCR}$} & yes & $56 \pm 15.0$ & $39.5 \pm 15.5$ & 8 & 7 & 8 & 7 & 5 & 10 & $13.3 \pm 5.7$ & $6.8 \pm 3.8$ \\
\hline & no & $48.9 \pm 13.6$ & $46.4 \pm 20.0$ & 4 & 10 & 12 & 2 & 11 & 3 & $9.9 \pm 5.6$ & $5.2 \pm 3.7$ \\
\hline & $p$ & 0.19 & 0.31 & 0.26 & & 0.11 & & 0.025 & & 0.11 & 0.38 \\
\hline
\end{tabular}

${ }^{a} p$ probability calculated using Fischer's Exact test on contingency tables (stage, ER IHC, PR IHC) or using t-test for comparison of age, tumor size, ERBB2/cell, and ERBB2/CEN17, as executed using JMP Statistical Software. All calculations use parameter cutoffs specified in Table 2

looked at the percentages of cells with greater than two gene or chromosome copies (gene or chromosome gain), or less than two copies (gene or chromosome loss), or the sum of these abnormal copy numbers (gene or chromosome gain or loss). This series of parameters is similar to those used in previous studies that compared different copy number parameters for associations with patient diagnoses and/or outcomes in melanoma [46], esophageal cancer [47], lung cancer [48], and cervical cancer [49]. This is the first application of these parameters in cases with neoadjuvant treatment of ERBB2positive breast cancer.

The importance of evaluating different parameters can be seen in Table 2 and Fig. 2 for the series of different parameters describing $M E T$ copy number relative to chromosome 7 copy number. In this series of parameters both gain of $M E T$ relative to chromosome 7 (AUC $=0.613$, sensitivity $=0.58$ and specificity $=0.67$ at the optimal cutoff of $32 \%$ cells with relative gain) and loss of $M E T$ relative to chromosome 7 (AUC $=0.651$, sensitivity $=0.50$ and specificity $=0.83$ at the optimal cutoff of $45 \%$ cells with relative loss) are associated weakly with $\mathrm{pCR}(p=0.41$ and 0.19 , respectively at the optimal cutoffs). However, the sum of cells with relative gain and loss (MET/CEN7 gain or loss) is highly associated with $\mathrm{pCR}$ (AUC $=0.791$, sensitivity = 0.92 and specificity $=0.67$ at the optimal cutoff of $50 \%$ cells with abnormal relative copy number with $p=0.0032$ ). Furthermore, the average ratio of $M E T / C E N 7$ has no association with $\mathrm{pCR}$, either high ratios or low ratios ( $p=1.0$ for either relationship at the optimal cutoffs), which is understandable since both $M E T / C E N 7$ gain (equating to higher ratio) and $M E T / C E N 7$ loss (equating to lower ratio) are associated with pCR and would tend to cancel each other in a ratio calculation. Therefore, proper parameter selection is very important since evaluation of these neoadjuvant specimens using a MET-to-CEN7 ratio, the most common measure of relative copy number, would have erroneously indicated a lack of prognostic value while use of the relative gene-to-chromosome imbalance parameter $M E T / C E N 7$ gain or loss provides a high statistical association. Similar to $M E T$ and chromosome 7, ISH data for the other genes and corresponding chromosomes were evaluated in terms of the various parameters defined in the Methods section of this paper. Of the other 3 gene probes only PTEN reached statistical significance and this was using PTEN gain in which patient tumors having higher numbers of cells with more than 2 copies of PTEN were associated with pCR (AUC $=0.674$, sensitivity $=0.38$ and specificity $=1.00$ at the optimal cutoff of $58 \%$ cells with PTEN gain, $p=0.039)$. Examining the actual ISH signals per cell and the average PTEN/CEN10 ratios, gain of PTEN was likely a result of chromosome 10 polysomy, with CEN10/cell ranging between 1.5 and 4.2 and PTEN/ CEN10 not exceeding a ratio of 1.4 for any one specimen. Several specimens did exhibit PTEN deletion $(P T E N / C E N 10<0.7)$ but these appeared in both the pCR and non-pCR groups and no association was found for deletion using the parameters of PTEN loss, PTEN/CEN10 ratio, or PTEN/CEN10 loss. 
Ki-67 expression, as measured by IHC, was also highly associated with $\mathrm{pCR}$ (AUC $=0.726$, sensitivity $=1.00$ and specificity $=0.42$ at the optimal cutoff of $8 \%$ cells expressing Ki-67, $p=0.0098$ ). The only Ki-67 parameter evaluated was the percentage of cells expressing $\mathrm{Ki}-67$, since this is a standard parameter used by pathologists in the evaluation of breast tissues. Since both $M E T / C E N 7$ gain or loss and Ki-67 parameters were highly associated with $\mathrm{pCR}$, the two were analyzed in combination by holding MET/CEN7 gain or loss at its optimal cutoff of $50 \%$ and varying the Ki-67 cutoff over a wide range (Fig. 2 and Table 2). This improved the AUC, and the optimal Ki-67 cutoff (8\% cells) provided sensitivity and specificity of $92 \%$ and $83 \%$, respectively, and reduced the $p$-value below either single parameter $(p=0.0006)$.

The association between $\mathrm{pCR}$ and MET/CEN7 gain or loss may be an important finding since common clinical and pathologic characteristics were not found to have statistically significant associations with pCR (Table 3) in the neoadjuvant setting tested here. Of interest, MET/ CEN7 gain or loss had little association with ERBB2 gene status but was associated with patient age and trended with tumor size and PR expression.

Our observations do not provide a mechanistic understanding of the role of MET copy number alterations in predicting $\mathrm{pCR}$ in HER2-positive breast cancer. And, these findings need to be validated in a subsequent, larger study of MET copy number alterations in a similar patient population. In an updated expansion cohort including patients treated with pertuzumab, additional studies with immunohistochemistry for MET and other markers to interrogate related signaling pathways (e.g., PI3K/AKT pathway) could be informative. One hypothesis is that any alteration of the MET signaling pathway may have some relationship to developing a pCR in the two treatment groups (ACTH and $\mathrm{TCH}$ ) analyzed in this study. Increased or decreased MET expression potentially resulting from MET gene copy number change might create a fragile condition sensitive to perturbation by therapeutics.

\section{Conclusions}

Our results show that a predictive score based on MET gene copy number relative to chromosome 7 and Ki-67 expression is strongly associated with $\mathrm{pCR}$ in patients with ERBB2-positive tumors treated with neoadjuvant chemotherapy and ERBB2-targeted therapy. Although the use of $\mathrm{pCR}$ as a surrogate endpoint for event free (EFS) and overall survival (OS) remains controversial, the ability to predict which patients are most likely to achieve a pCR remains important for individual patient treatment decisions and future clinical trial design. Patients with a sufficiently low likelihood of a $\mathrm{pCR}$ who are not candidates for breast conservation at presentation may choose surgery followed by adjuvant chemotherapy and ERBB2-targeted therapy. These data from retrospective studies require validation in a larger, prospective study.

\section{Additional file}

Additional file 1: Supplementary Data - In situ hybridization parameters calculated for individual patients as used for ROC and contingency analyses (Tables 2 and 3 and Figs. 2 and 3 in main article). (XLS 57 kb)

\section{Acknowledgements}

Not applicable.

\section{Funding}

Funding was provided for the Cleveland Clinic authors by the Cleveland Clinic Foundation. Funding was provided for the Ventana author by Ventana Medical Systems, Inc., which also provided ISH and IHC reagents for this study. The funders had no other role other than internal review board approval (Cleveland Clinic) and approval of the final manuscript for publication.

Availability of data and materials

Data generated and analyzed during this study are included in this published article and its supplementary information file [Additional file 1].

\section{Authors' contributions}

BC collected clinical and pathological data, prepared of tables and figures, and participated in manuscript drafting and revision. BP collected pathology data, provided technical support, and participated in manuscript revision. ZW provided technical support. EM collected clinical data and participated in manuscript revision. TB contributed to conception and design of the study, and collected and analyzed clinical data. $\mathrm{CL}$ performed IHC and ISH tissue staining, and participated in data acquisition and manuscript review. RT contributed experimental design, project oversight, and pathologic evaluation of $\mathrm{IHC}$ and ISH staining. LM performed ISH and IHC data analysis for parameter associations with $\mathrm{pCR}$, prepared tables and figures, and participated in manuscript drafting and revision. We sadly report that RT died prior to manuscript preparation. All other authors read and approved the manuscript.

\section{Competing interests}

LM declares he is an employee of Ventana Medical Systems Inc., a diagnostics company in the Roche Group, and owns stocks and options to purchase stocks in the Roche Group. BP declares he had no competing interests at the time this work was performed, but is now an employee of Ventana Medical System, Inc., a diagnostics company in the Roche Group. All other authors declare they have no competing interests.

Consent for publication

Not applicable.

\section{Ethics approval and consent to participate}

This study, including the chart review and the collection, storage and analysis of de-identified data was approved by the Cleveland Clinic Institutional Review Board, which also waived the need for informed consent to participate in the study.

\section{Author details}

1Department of Pathology, Cleveland Clinic, 9500 Euclid Avenue, Cleveland, $\mathrm{OH}$ 44195, USA. ${ }^{2}$ Department of Hematology and Oncology, Cleveland Clinic, Cleveland, OH, USA. ${ }^{3}$ Present Address: Ventana Medical Systems, Inc, 1910 E. Innovation Park Dr, Tucson, AZ 85755, USA.

Received: 6 January 2016 Accepted: 22 August 2016

Published online: 30 August 2016 


\section{References}

1. Cortazar P, Zhang L, Untch M, Mehta K, Costantino JP, Wolmark N, et al. Pathological complete response and long-term clinical benefit in breast cancer: the CTNeoBC pooled analysis. Lancet. 2014;384:164-72.

2. Guidance for Industry Pathological Complete Response in Neoadjuvant Treatment of High-Risk Early-Stage Breast Cancer: Use as an Endpoint to Support Accelerated Approval. U.S. Department of Health and Human Services; Food and Drug Administration; Center for Drug Evaluation and Research (CDER). 2014. http://www.fda.gov/downloads/drugs/guidancecompliance regulatoryinformation/guidances/ucm305501.pdf. Accessed 6 June 2015.

3. Gianni L, Pienkowski T, Im YH, Roman L, Tseng LM, Liu MC, et al. Efficacy and safety of neoadjuvant pertuzumab and trastuzumab in women with locally advanced, inflammatory, or early HER2-positive breast cancer (NeoSphere): a randomised multicentre, open-label, phase 2 trial. Lancet Oncol. 2012;13:25-32.

4. Cancer Genome Atlas N. Comprehensive molecular portraits of human breast tumours. Nature. 2012;490:61-70.

5. Curtis C, Shah SP, Chin SF, Turashvili G, Rueda OM, Dunning MJ, et al. The genomic and transcriptomic architecture of 2,000 breast tumours reveals novel subgroups. Nature. 2012;486:346-52.

6. Berns K, Horlings HM, Hennessy BT, Madiredjo M, Hijmans EM, Beelen K, et al. A functional genetic approach identifies the PI3K pathway as a major determinant of trastuzumab resistance in breast cancer. Cancer Cell. 2007; 12:395-402.

7. Junttila T, Akita RW, Parsons K, Fields C, Lewis Phillips GD, Friedman LS, et al. Ligand-independent HER2/HER3/PI3K complex is disrupted by trastuzumab and is effectively inhibited by the PI3K inhibitor GDC-0941. Cancer Cell. 2009;15:429-40.

8. Kataoka Y, Mukohara T, Shimada H, Saijo N, Hirai M, Minami H. Association between gain-of-function mutations in PIK3CA and resistance to HER2targeted agents in HER2-amplified breast cancer cell lines. Ann Oncol. 2010;21:255-62

9. Eichhorn PJ, Gili M, Scaltriti M, Serra V, Guzman M, Nijkamp W, et al. Phosphatidylinositol 3-kinase hyperactivation results in lapatinib resistance that is reversed by the mTOR/phosphatidylinositol 3-kinase inhibitor NVPBEZ235. Cancer Res. 2008:68:9221-30.

10. Loi S, Michiels S, Lambrechts D, Fumagalli D, Claes B, Kellokumpu-Lehtinen PL, et al. Somatic mutation profiling and associations with prognosis and trastuzumab benefit in early breast cancer. J Natl Cancer Inst. 2013;105:960-7.

11. Pogue-Geile KL, Song N, Jeong JH, Gavin PG, Kim SR, Blackmon NL, et al. Intrinsic subtypes, PIK3CA mutation, and the degree of benefit from adjuvant trastuzumab in the NSABP B-31 trial. J Clin Oncol. 2015;33:1340-7.

12. Majewski IJ, Nuciforo P, Mittempergher L, Bosma AJ, Eidtmann H, Holmes $E_{\text {, }}$ et al. PIK3CA mutations are associated with decreased benefit to neoadjuvant human epidermal growth factor receptor 2-targeted therapies in breast cancer. J Clin Oncol. 2015;33:1334-9.

13. Schneeweiss A, Chia S, Hegg R, Tausch C, Deb R, Ratnayake J, et al. Evaluating the predictive value of biomarkers for efficacy outcomes in response to pertuzumab- and trastuzumab-based therapy: an exploratory analysis of the TRYPHAENA study. Breast Cancer Res. 2014;16:R73.

14. Loibl S, von Minckwitz G, Schneeweiss A, Paepke S, Lehmann A, Rezai M, et al. PIK3CA mutations are associated with lower rates of pathologic complete response to anti-human epidermal growth factor receptor 2 (her2) therapy in primary HER2-overexpressing breast cancer. J Clin Oncol. 2014;32:3212-20.

15. Firoozinia M, Zareian Jahromi M, Moghadamtousi SZ, Nikzad S, Abdul KH. PIK3CA gene amplification and PI3K p110alpha protein expression in breast carcinoma. Int J Med Sci. 2014;11:620-5.

16. Huw LY, O'Brien C, Pandita A, Mohan S, Spoerke JM, Lu S, et al. Acquired PIK3CA amplification causes resistance to selective phosphoinositide 3kinase inhibitors in breast cancer. Oncogenesis. 2013;2:e83.

17. Li J, Yen C, Liaw D, Podsypanina K, Bose S, Wang SI, et al. PTEN, a putative protein tyrosine phosphatase gene mutated in human brain, breast, and prostate cancer. Science. 1997;275:1943-7.

18. Zhang S, Yu D. PI(3)king apart PTEN's role in cancer. Clin Cancer Res. 2010; 16:4325-30.

19. Nagata $Y$, Lan KH, Zhou X, Tan M, Esteva FJ, Sahin AA, et al. PTEN activation contributes to tumor inhibition by trastuzumab, and loss of PTEN predicts trastuzumab resistance in patients. Cancer Cell. 2004;6:117-27.

20. Fujita T, Doihara H, Kawasaki K, Takabatake D, Takahashi H, Washio K, et al. PTEN activity could be a predictive marker of trastuzumab efficacy in the treatment of ErbB2-overexpressing breast cancer. Br J Cancer. 2006;94:247-52.
21. Faratian D, Goltsov A, Lebedeva G, Sorokin A, Moodie S, Mullen P, et al. Systems biology reveals new strategies for personalizing cancer medicine and confirms the role of PTEN in resistance to trastuzumab. Cancer Res. 2009;69:6713-20.

22. Dave B, Migliaccio I, Gutierrez MC, Wu MF, Chamness GC, Wong H, et al. Loss of phosphatase and tensin homolog or phosphoinositol-3 kinase activation and response to trastuzumab or lapatinib in human epidermal growth factor receptor 2-overexpressing locally advanced breast cancers. J Clin Oncol. 2011;29:166-73.

23. Esteva FJ, Guo H, Zhang S, Santa-Maria C, Stone S, Lanchbury JS, et al. PTEN, PIK3CA, p-AKT, and p-p70S6K status: association with trastuzumab response and survival in patients with HER2-positive metastatic breast cancer. Am J Pathol. 2010;177:1647-56.

24. Razis E, Bobos M, Kotoula V, Eleftheraki AG, Kalofonos HP, Pavlakis K, et al. Evaluation of the association of PIK3CA mutations and PTEN loss with efficacy of trastuzumab therapy in metastatic breast cancer. Breast Cancer Res Treat. 2011;128:447-56.

25. Perez EA, Dueck AC, McCullough AE, Chen B, Geiger XJ, Jenkins RB, et al. Impact of PTEN protein expression on benefit from adjuvant trastuzumab in early-stage human epidermal growth factor receptor 2-positive breast cancer in the North Central Cancer Treatment Group N9831 trial. J Clin Oncol. 2013;31:2115-22.

26. Smith K, Houlbrook S, Greenall M, Carmichael J, Harris AL. Topoisomerase II alpha co-amplification with erbB2 in human primary breast cancer and breast cancer cell lines: relationship to m-AMSA and mitoxantrone sensitivity. Oncogene. 1993;8:933-8.

27. Hoare SF, Freeman CA, Coutts JC, Varley JM, James L, Keith WN. Identification of genetic changes associated with drug resistance by reverse in situ hybridization. Br J Cancer. 1997;75:275-82.

28. Keith WN, Douglas F, Wishart GC, McCallum HM, George WD, Kaye SB, et al. Co-amplification of erbB2, topoisomerase II alpha and retinoic acid receptor alpha genes in breast cancer and allelic loss at topoisomerase I on chromosome 20. Eur J Cancer. 1993;29A:1469-75.

29. Murphy DS, McHardy P, Coutts J, Mallon EA, George WD, Kaye SB, et al. Interphase cytogenetic analysis of erbB2 and topoll alpha co-amplification in invasive breast cancer and polysomy of chromosome 17 in ductal carcinoma in situ. Int J Cancer. 1995;64:18-26.

30. Press MF, Sauter G, Buyse M, Bernstein L, Guzman R, Santiago A, et al. Alteration of topoisomerase II-alpha gene in human breast cancer: association with responsiveness to anthracycline-based chemotherapy. J Clin Oncol. 2011;29:859-67.

31. Fasching PA, Weihbrecht S, Haeberle L, Gasparyan A, Villalobos IE, Ma Y, et al. HER2 and TOP2A amplification in a hospital-based cohort of breast cancer patients: associations with patient and tumor characteristics. Breast Cancer Res Treat. 2014:145:193-203.

32. Park K, Han S, Gwak GH, Kim HJ, Kim J, Kim KM. Topoisomerase II-alpha gene deletion is not frequent as its amplification in breast cancer. Breast Cancer Res Treat. 2006;98:337-42.

33. Park K, Kim J, Lim S, Han S. Topoisomerase II-alpha (topoll) and HER2 amplification in breast cancers and response to preoperative doxorubicin chemotherapy. Eur J Cancer. 2003;39:631-4.

34. Lengyel E, Prechtel D, Resau JH, Gauger K, Welk A, Lindemann K, et al. C-Met overexpression in node-positive breast cancer identifies patients with poor clinical outcome independent of Her2/neu. Int J Cancer. 2005; 113:678-82

35. Ghoussoub RA, Dillon DA, D'Aquila T, Rimm EB, Fearon ER, Rimm DL. Expression of c-met is a strong independent prognostic factor in breast carcinoma. Cancer. 1998:82:1513-20.

36. Camp RL, Rimm EB, Rimm DL. Met expression is associated with poor outcome in patients with axillary lymph node negative breast carcinoma. Cancer. 1999:86:2259-65

37. Tolgay Ocal I, Dolled-Filhart M, D'Aquila TG, Camp RL, Rimm DL. Tissue microarray-based studies of patients with lymph node negative breast carcinoma show that met expression is associated with worse outcome but is not correlated with epidermal growth factor family receptors. Cancer. 2003:97:1841-8

38. Kang JY, Dolled-Filhart M, Ocal IT, Singh B, Lin CY, Dickson RB, et al. Tissue microarray analysis of hepatocyte growth factor/Met pathway components reveals a role for Met, matriptase, and hepatocyte growth factor activator inhibitor 1 in the progression of node-negative breast cancer. Cancer Res. 2003:63:1101-5. 
39. Chen HH, Su WC, Lin PW, Guo HR, Lee WY. Hypoxia-inducible factor-1alpha correlates with MET and metastasis in node-negative breast cancer. Breast Cancer Res Treat. 2007;103:167-75.

40. Ho-Yen CM, Green AR, Rakha EA, Brentnall AR, Ellis IO, Kermorgant S, et al. C-Met in invasive breast cancer: is there a relationship with the basal-like subtype? Cancer. 2014;120:163-71.

41. Minuti G, Cappuzzo F, Duchnowska R, Jassem J, Fabi A, O'Brien T, et al. Increased MET and HGF gene copy numbers are associated with trastuzumab failure in HER2-positive metastatic breast cancer. Br J Cancer. 2012;107:793-9.

42. Ho-Yen $\mathrm{CM}$, Jones JL, Kermorgant $\mathrm{S}$. The clinical and functional significance of c-Met in breast cancer: a review. Breast Cancer Res. 2015;17:52.

43. Wolff AC, Hammond ME, Schwartz JN, Hagerty KL, Allred DC, Cote RJ, et al. American Society of Clinical Oncology/College of American Pathologists guideline recommendations for human epidermal growth factor receptor 2 testing in breast cancer. J Clin Oncol. 2007:25:118-45.

44. Shultz EK. Multivariate receiver-operating characteristic curve analysis: prostate cancer screening as an example. Clin Chem. 1995:41:1248-55.

45. Pauletti G, Dandekar S, Rong H, Ramos L, Peng H, Seshadri R, et al. Assessment of methods for tissue-based detection of the HER-2/neu alteration in human breast cancer: a direct comparison of fluorescence in situ hybridization and immunohistochemistry. J Clin Oncol. 2000;18:3651-64.

46. Gerami P, Jewell SS, Morrison LE, Blondin B, Schulz J, Ruffalo T, et al. Fluorescence in situ hybridization (FISH) as an ancillary diagnostic tool in the diagnosis of melanoma. Am J Surg Pathol. 2009:33:1146-56.

47. Geppert Cl, Rummele P, Sarbia M, Langer R, Feith M, Morrison L, et al. Multi-colour FISH in oesophageal adenocarcinoma-predictors of prognosis independent of stage and grade. Br J Cancer. 2014;110:2985-95.

48. Fidler MJ, Morrison LE, Basu S, Buckingham L, Walters K, Batus M, et al. PTEN and PIK3CA gene copy numbers and poor outcomes in non-small cell lung cancer patients with gefitinib therapy. Br J Cancer. 2011;105:1920-6.

49. Heselmeyer-Haddad K, Janz V, Castle PE, Chaudhri N, White N, Wilber K, et al. Detection of genomic amplification of the human telomerase gene (TERC) in cytologic specimens as a genetic test for the diagnosis of cervical dysplasia. Am J Pathol. 2003;163:1405-16.

\section{Submit your next manuscript to BioMed Central and we will help you at every step:}

- We accept pre-submission inquiries

- Our selector tool helps you to find the most relevant journal

- We provide round the clock customer support

- Convenient online submission

- Thorough peer review

- Inclusion in PubMed and all major indexing services

- Maximum visibility for your research

Submit your manuscript at www.biomedcentral.com/submit

) Biomed Central 